\title{
THE EX ANTE INCENTIVE COMPATIBLE CORE IN THE ABSENCE OF WEALTH EFFECTS
}

\author{
By Françoise Forges, Jean-François Mertens, \\ AND RAJIV VOHRA ${ }^{1}$
}

In a differential information economy with quasi-linear utilities, monetary transfers facilitate the fulfillment of incentive compatibility constraints: the associated ex ante core is generically non-empty. However, we exhibit a well-behaved exchange economy in which this core is empty, even if goods are allocated through random mechanisms.

KEYWORDS: Core, exchange economy, asymmetric information, incentive compatibility, ex ante, transferable utility, absence of wealth effects, quasi-linear.

\section{INTRODUCTION}

CONSIDER AN EXCHANGE ECONOMY in which consumers have private information (represented by their types). Issues of resource allocation in such an economy concern how the allocation is determined by the information state (profile of agents' types). An allocation rule, in which the (possibly random) allocation depends on the information state of the economy, can then be viewed as a direct mechanism, representing some unspecified indirect mechanism (that might include auctions, boycotts, etc.). As is well explained in Holmström and Myerson (1983), there is no unique and obvious way of extending the notion of Pareto efficiency to mechanisms of such an economy. Naturally, this observation also applies to the notion of the core - which is the primary interest of the present paper. An appropriate core notion depends on whether decisions are made at the ex ante stage or the interim stage.

Defining a core concept at the interim stage involves conceptual difficulties because even the player set is not clear (one player per type?), let alone the concept of characteristic function. Wilson himself, in his seminal paper Wilson (1978), proposes two different possibilities, the coarse core and the fine core. And several other core notions too have

\footnotetext{
${ }^{1}$ We wish to thank Enrico Minelli for numerous, extremely useful discussions on the topic of this research. Also conversations with Claude d'Aspremont, Andy Postlewaite, Roy Radner, Debraj Ray and Roberto Serrano and anonymous referees' comments have been very helpful. Financial support from European TMR Network FMRX CT 960055 and from a Salomon research grant, Brown University, is gratefully acknowledged.
} 
been analyzed for differential information economies at the interim stage. $^{2}$ But at the ex ante stage, there is no ambiguity whatsoever about the correct characteristic function, as we will argue in section 2 . Thus a full cooperative analysis for such exchange economies can be performed by applying any standard solution concept, like the core, to their characteristic function.

Our main concern in this paper is this ex ante incentive compatible core: coalitions can agree, ex ante, upon any incentive compatible mechanism, where a mechanism goes from a set of profiles of players' actions (sending orders, giving collateral, etc.) to the set of outcomes, i.e., the feasible set. $V(S)$ describes then the set of expected utility vectors that $S$ can get for its members by any such mechanism. Coalition $S$ has an objection to some mechanism if it can find an incentive compatible mechanism (for $S$ ) that increases ${ }^{3}$ the expected utility of each agent in $S$. The ex ante incentive compatible core is the set of all incentive compatible mechanisms to which there exists no objection. It is the core analog of Holmström and Myerson (1983)'s notion of ex ante incentive efficiency. A similar concept was used in Allen (1992, 1994) and Vohra (1999). ${ }^{4}$

It is worth emphasizing that (with concave utilities), when incentive constraints are not imposed, the ex ante characteristic function is, for the grand coalition, the same as for the Arrow-Debreu economy with a complete set of contingent commodities, and for sub-coalitions, included in the latter. So this characteristic function has a non-empty core, and since the same holds for the economy restricted to any subset of players, it is totally balanced. In particular, with quasi-linear utilities, it is the characteristic function of some pure-exchange economy, by the well-known result of Shapley and Shubik (1969). Not surprisingly, incentive constraints make a significant difference to the analysis, and the question of non-emptiness becomes much more elusive. Vohra (1999) identifies some cases where the ex ante core ${ }^{5}$ is non-empty: two-

\footnotetext{
${ }^{2}$ For example, Ichiishi and Idzik (1996), Lee and Volij (2000), Vohra (1999), Volij (2000), Yannelis (1991). See Forges, Minelli and Vohra (2001) for additional references.

${ }^{3}$ Given the absence of wealth effects, no weak- vs. strict core distinction arises.

${ }^{4}$ Even for the ex ante stage there is an extensive literature which follows a different approach. For example, Allen (1993), Koutsougeras and Yannelis (1993), Yannelis (1991), impose various forms of measurability constraints on trades instead of incentive constraints. We refer the reader to Forges, Minelli and Vohra (2001) for a discussion.

${ }^{5}$ Henceforth when we use the term 'ex ante core' without qualification it should be taken to refer to the ex ante incentive compatible core.
} 
consumer economies, economies with linear utility, and economies with a non-exclusive information structure. McLean and Postlewaite (1999, 2000) introduce the notion of an economy with informationally small agents and prove that in such an economy, the ex ante core is nonempty.

We show here that non-emptiness does depend on such additional assumptions. Already, restricting attention to deterministic mechanisms, Vohra (1999) provides an example of a well-behaved economy in which the ex ante core is empty. Our aim in the present paper is to investigate the extent to which this problem can be solved by allowing for random mechanisms and monetary transfers, both of which are generally known to be of help in fulfilling incentive constraints.

In fact, Allen (1992) obtains a non-empty ex ante core with randomization, but with feasibility in expected terms, à la Prescott and Townsend (1984). The question then is whether this remains true with exact feasibility, as required by mechanisms. And recent results of Forges and Minelli (1999) suggest that randomization indeed does make a difference: the ex ante core is then not empty in the example of Vohra (1999); moreover, this holds more generally, as long as no commodity is initially owned by more than one agent, and utility functions are additively separable across goods. So randomization cannot be neglected in investigating the ex ante core.

It is also well known that the possibility of monetary transfers can facilitate the satisfaction of incentive constraints. An extensive literature deals indeed with conditions for transfer schemes to exist that achieve both incentive compatibility and first-best efficiency; see in particular, Arrow (1979), d'Aspremont and Gérard-Varet (1979, 1982), d'Aspremont, Crémer and Gérard-Varet (1990, 1992), Crémer and McLean (1985), Green and Laffont (1979), Groves (1973), Groves and Loeb (1975), Johnson, Pratt and Zeckhauser (1990). This is the motivation for studying the ex ante core in a quasi-linear setup.

In section 4 we provide our main positive result, that generically (in endowments) the ex ante core is non-empty. We also show how other results from the mechanism design literature can be fruitfully applied to the present problem, and yield generic non-emptiness of the ex ante core. In particular, several conditions for Bayesian implementation of first-best mechanisms (such as independent private values) also serve to ensure non-emptiness. The argument actually proceeds by showing that a mechanism in the ex ante core without incentive constraints can be modified with appropriate transfers to ensure incentive compatibility, leaving unchanged the ex ante utility levels. We have therefore, in addition to those in Vohra (1999), Forges and Minelli (1999) and 
McLean and Postlewaite (2000), several cases in which the ex ante core is non-empty.

Unfortunately, as we shall show in section 5, extending thereby the negative conclusion of Vohra (1999), such positive results must remain generic: we provide a robust example of an economy with quasi-linear utilities where the ex ante core is empty. This is so even though we permit the grand coalition to use lotteries and free disposal of all goods including money, while the sub-coalitions use none of those facilities.

In section 6 we indicate why this implies a fortiori the same negative result for standard Walrasian economies (i.e., pure exchange, with the non-negative orthant as consumption set). Moreover, our example can be modified to show that our main positive result does not extend to economies in which monetary transfers are bounded.

In section 7 an interim concept, the incentive compatible version (Vohra (1999)) of Wilson (1978)'s coarse core, is shown to be empty too in the example.

In the appendix we provide results on the continuity of the characteristic function with respect to the underlying economy. Those are used in section 5 to demonstrate the robustness of our example.

\section{Formulation of the Problem}

2.1. Private Information. Each agent's private information is represented by his type, and an information state refers to a profile of agents' types. The beliefs of the agents are described by a probability distribution on the product of the sets of types. There is an alternative framework in which uncertainty concerns a state of the world, and private information is described by a partition of this state space. This can be translated into the framework with types simply by associating each element of an agent's information partition with a particular type. This construction may obviously generate type vectors of probability zero, but the two models are equivalent. However, types become necessary as soon as one wants to define incentive compatible mechanisms. Indeed, a mechanism should not be defined over the "true" states of the world but over the types that are reported by the agents; there is no way to prevent these independent reports from being 'inconsistent', and yet the mechanism has to be defined on such reports. Of course, on type vectors that have zero probability, a mechanism may allocate goods in a special way (e.g., confiscating the agents' endowments).

2.2. Endowments. In general, an agent's endowments may depend on the information state. We assume that once an agent learns his type he also knows his endowment. This is not an ad hoc assumption; in 
any exchange model, agents have to be able to put their hands on their endowment at the time of exchange. That is the relevant time for the interim stage: when they have obtained their full private information, including complete information about their endowment.

2.3. Preferences. The preferences of an agent may depend, in principle, on the entire profile of types; an agent's type may indeed include information that is relevant to another agent (common values). We assume that preferences are represented by a von-Neumann-Morgenstern utility function. At the ex ante (resp. interim) stage, one thus measures an agent's utility in terms of his expected utility (resp. expected utility conditional on his type).

2.4. The characteristic function. The core concept rests on that of a characteristic function, and hence is applicable only to games that are "well represented" by their characteristic function ("fixed threat games", or "c-games" in Shubik's terminology, e.g., Shubik (1982) Chapter $6, \S 2$, and references there). ${ }^{6}$

And at the ex ante stage, economies with differential information are c-games, just as in the complete information case: coalition $S$ can even simultaneously guarantee any point in $V(S)$ and prevent $\complement S$ to obtain any point outside $V(\complement S)$ (in particular, $V$ also equals $V_{\alpha}$ ), just by refusing to give any goods or information away to $\complement S$, and implementing her own mechanism: the game is "well represented by $V$ " in the strongest possible (much stronger than fn. 6) and most obvious sense. Thus a full cooperative analysis for such economies can be performed by applying any standard solution concept to their characteristic function $V$. The only problem is thus to ascertain the correct characteristic function, i.e., what utility vectors a coalition can guarantee herself, by any means.

To take into account the differential information aspects, those means have to be construed as mechanisms: coalitions can agree, ex ante, upon any incentive compatible mechanism — where a mechanism maps the profiles of players' actions (sending orders, giving collateral, etc.) to the set of outcomes, i.e., the feasible set. $V(S)$ describes the set of expected utility vectors that $S$ can get for its members by any such mechanism. Observe that this definition directly implies that $V(S)$

\footnotetext{
${ }^{6} \mathrm{~A}$ minimal operational meaning of fixed threats in a strategic game is that the worth $v_{\lambda}(S)$ (in the Harsanyi-Shapley variable threat solution) of a coalition $S$ as a function of the vector $\lambda$ of utility-weights can be obtained as $\max _{u_{S} \in V(S)}\left\langle\lambda, u_{S}\right\rangle-$ $\max _{u_{\mathcal{C} S} \in V(\mathcal{C} S)}\left\langle\lambda, u_{\complement S}\right\rangle$, for appropriate sets $V(S)$ and $V(\complement S)$. Those should be taken as convex, since in this paradigm coalitions randomize. In particular, $V$ is then a $\beta$-characteristic function. The criterion amounts to ask that $v_{\lambda}(S)$ be additively separable as the sum of a convex function of $\lambda_{\mid S}$ and a concave function of $\lambda_{\mid C S}$.
} 
is convex-valued and superadditive, hence a meaningful characteristic function.

2.5. The class of mechanisms. We need thus a class of mechanisms sufficiently wide as to capture all potentially relevant ("any") means.

A first aspect is standard (e.g., in Myerson (1984)'s original definition): to allow for randomizing mechanisms (thus ensuring the convexity of the set of incentive compatible mechanisms). Not only is it part of the very definition of mechanisms, so one should dispense with it only for models where one can prove it is immaterial, e.g. standard exchange economies, but it is also required for the validity of the revelation principle itself, which we definitely do use by restricting attention throughout to direct mechanisms.

In our context, we mentioned above an example where it made a critical difference; so certainly we cannot omit this aspect. That example was in a Walrasian framework; without wealth effects, such phenomena are very familiar too, e.g. from buyer-seller problems for an indivisible object (with finite type sets). For an example falling strictly within our framework of quasi-linear exchange economies, consider two agents, and two equally likely states, agent 1 being informed and agent 2 not. There is a single commodity, with endowment $(1,1)$ in each state. Utilities in the first state are $x$ for agent 1 and $1.25 x$ for agent 2 , and in the other state, $x /(x+1)$ for 1 and 0 for $2 .^{7}$

A second aspect relates to free disposal, often taken as innocuous in a complete information setup. We intend to take it seriously: the implied feasible set means that mechanisms can throw away goods (including money). In our framework of differential information, this might substantially facilitate the fulfillment of incentive constraints. For an example, take a one commodity economy with two agents, each one having two types; the high type has utility $2 x$ and the low, $x$. The probability of both agents being high is $1 / 7$; else their types are opposite, both cases equally likely. A unit quantity of the commodity is available. ${ }^{8}$

\footnotetext{
${ }^{7}$ Then the endowment is the optimal deterministic incentive compatible mechanism, while (ex-post) efficiency requires $(0,2),(2,0)$. Replace now the allocation in the first state by a randomization between giving everything to 1 and giving everything to 2: expected utilities are the same, and the expected utility for 1 of lying has decreased in the second state, and remained the same in the first state. One can infer from this that, at this new, equivalent mechanism, the incentive constraints are no longer binding (cf. (14) below), so it can be perturbed a bit towards the first best while remaining incentive-compatible: one achieves strictly more with incentive compatible mechanisms by randomization.

${ }^{8}$ Consider the mechanism which gives everything to the high type when the other is of low type, which confiscates everything when both claim to be high, and else 
The last aspect deals with variability of endowments. In that case, mechanisms where agents would just "report their types" would face major feasibility issues concerning the implied net trades when agents misreport. Such issues are solved in practice in the obvious way: by the use of various forms of collateral. Thus mechanisms ought to be able to require traders to deposit appropriate collateral for each order, in order to ensure feasibility of any implied net trade, as, for example, in Hurwicz, Maskin and Postlewaite (1995).

This leads one to consider mechanisms in which each agent is asked to report his type as well as his endowment. By applying the revelation principle to this class of mechanisms, one can assume, without loss of generality, that an agent reports just a type (recall that an agent's endowment depends only on his type). In particular, a report of a type is also (implicitly) a statement about the endowment. Since endowments cannot be over-reported, types are partially verifiable.

\section{THE MODEL}

The exchange economy involves $n$ agents, $l$ consumption goods and money. Agent $i$ 's private information is represented by his type $t^{i} \in T^{i}$ $(i=1, \ldots, n)$, where $T^{i}$ is a finite set. Let us define $N=\{1, \ldots, n\}$ and $T=\prod_{i=1}^{n} T^{i}$. Let $q$ be the prior probability distribution over $T$; w.l.o.g., we assume that $q\left(t^{i}\right)>0, \forall t^{i} \in T^{i}$. ${ }^{9}$ Agent $i$ 's initial endowment $e^{i} \in \mathbb{R}_{+}^{l}$ depends only on his own type. His preferences are represented by a quasi-linear von Neumann-Morgenstern utility function

$$
u^{i}: T \times \mathbb{R}_{+}^{l} \times \mathbb{R} \rightarrow \mathbb{R}:\left(t, x^{i}, m^{i}\right) \mapsto u^{i}\left(t, x^{i}, m^{i}\right)=w^{i}\left(t, x^{i}\right)+m^{i}
$$

where $\forall t \in T, w^{i}(t,$.$) is continuous, concave and increasing. Thus, an$ agent's preferences can depend on the others' information.

We shall associate a cooperative game, i.e., a characteristic function, with the economy. A coalition $S$ is a (non-empty) subset of $N$. The set of actions of agent $i$ is a copy $A^{i}$ of $T^{i}$, and we use

$$
A_{S}=\prod_{i \in S} A^{i} \quad T_{S}=\prod_{i \in S} T^{i}
$$

splits equally. It is incentive compatible, and yields $6 / 7$ as expected utility to each. And any incentive compatible mechanism that does not use free disposal yields a sum of expected utilities less than 12/7: by summing both low types' incentive constraints, the sum $x$ of their consumptions, when their opponent is high, is at least 1 ; next, again since free disposal is disallowed, expected total utility equals $2-\frac{3}{7} x$.

${ }^{9}$ The model is fully robust to the addition of zero probability types, because, as will be clear below, a monetary penalty can be imposed to any agent who would claim to be of such a type. 
It is understood that action $a_{0}^{i}$ of $i$ consists of claiming to be of type $a_{0}^{i}$ and depositing $e^{i}\left(a_{0}^{i}\right)$ as collateral. Hence it is available to $i$ only when of a type $t^{i}$ such that $e^{i}\left(t^{i}\right) \geq e^{i}\left(a_{0}^{i}\right) . t_{S}$ (resp. $\left.a_{S}\right)$ denotes a typical element $\left(t^{i}\right)_{i \in S}$ of $T_{S}$ (resp. $\left(a^{i}\right)_{i \in S}$ of $\left.A_{S}\right)$. Let us define, $\forall a \in A_{S}$,

$$
X_{S}(a)=\left\{x=\left(x^{i}\right)_{i \in S} \in\left(\mathbb{R}_{+}^{l}\right)^{S} \mid \sum_{i \in S} x^{i} \leq \sum_{i \in S} e^{i}\left(a^{i}\right)\right\}
$$

and let $\Delta(Y)$ be the set of probability distributions over (the Borel sets of) $Y$. A mechanism for coalition $S$ is a pair $\left(\xi_{S}, \mu_{S}\right)$ of maps s.t.

$$
\begin{gathered}
\xi_{S}(\cdot \mid a)=\xi_{S}(a) \in \Delta\left(X_{S}(a)\right), \quad \forall a \in A_{S} \\
\mu_{S}: A_{S} \rightarrow \mathbb{R}^{S}: a \mapsto\left(\mu_{S}^{i}(a)\right)_{i \in S}, \quad \text { with } \sum_{i \in S} \mu_{S}^{i}(a) \leq 0 \quad \forall a \in A_{S}
\end{gathered}
$$

The mechanism $\xi_{S}$ on consumption goods consists of a type dependent lottery, while money transfers are w.l.o.g. deterministic: as we shall see below, random money transfers would generate the same characteristic function. Feasibility requires that any allocation selected by $\xi_{S}$ be feasible, i.e., in $X_{S}(\cdot)$. Some of our results will hold for deterministic mechanisms $\xi_{S}$, in which for every $a$, the lottery $\xi_{S}(\cdot \mid a)$ is degenerate on a single allocation (i.e., $\xi_{S}$ is just a map to $\left.X_{S}(\cdot)\right)$.

Ex ante cooperative analysis means that agents contract before obtaining their private information, and report this information after, in order to execute those contracts. This is the relevant timing structure not only when designing institutions or examining their optimality, "behind the veil of ignorance", but e.g. also for R\&D cooperation contracts between firms, or for insurance contracts as in Prescott and Townsend $(1984$, sect. $\dot{6})$.

More precisely, we think of the following scenario:

- "Bargaining stage": a coalition may "form", in the usual sense of cooperative theory.

- Nature chooses $t=\left(t^{1}, \ldots, t^{n}\right)$ in $T$ according to $q$; each agent $i$ is only informed of his own type $t^{i}$.

- If $S$ has formed, every member $i$ of $S$ must report a type $a^{i} \in A^{i}$.

- If $\left(\xi_{S}, \mu_{S}\right)$ is the mechanism for $S$, every agent $i \in S$ receives the bundle $x^{i}$, where $\left(x^{i}\right)_{i \in S}$ is selected according to the probability distribution $\xi_{S}\left(\cdot \mid a_{S}\right)$, and the money transfer $\mu_{S}^{i}\left(a_{S}\right)$.

The mechanism $\left(\xi_{S}, \mu_{S}\right)$ is incentive compatible if the truthful strategies (i.e., $a^{i}=t^{i}$ for every agent $i \in S$ ) form a Nash equilibrium of the game induced by the above scenario.

The expected utility of agent $i \in S$, of type $t^{i}$, is, when reporting $a^{i}$ $U_{\xi_{S}, \mu_{S}}^{i}\left(t^{i}, a^{i}\right)=\sum_{t^{-i}} q\left(t^{-i} \mid t^{i}\right)\left[\mu_{S}^{i}\left(a^{i}, t_{S \backslash\{i\}}\right)+\int_{\mathbb{R}_{+}^{l}} w^{i}\left(t, y^{i}\right) \xi_{S}\left(d x^{i} \mid a^{i}, t_{S \backslash\{i\}}\right)\right]$ 
where $t^{-i}=t_{\mathrm{C}\{i\}}$, and $y^{i}=e^{i}\left(t^{i}\right)-e^{i}\left(a^{i}\right)+x^{i}$. When $a^{i}=t^{i}$, we write

$$
U_{\xi_{S}, \mu_{S}}^{i}\left(t^{i}\right)=U_{\xi_{S}, \mu_{S}}^{i}\left(t^{i}, t^{i}\right) \text {. }
$$

$\left(\xi_{S}, \mu_{S}\right)$ is incentive compatible iff

$$
U_{\xi_{S}, \mu_{S}}^{i}\left(t^{i}\right) \geq U_{\xi_{S}, \mu_{S}}^{i}\left(t^{i}, a^{i}\right) \quad \forall i \in S, \forall t^{i}, \forall a^{i} \in A^{i} \text { available to } t^{i}
$$

Observe that the incentive compatibility constraints that would be derived from general random mechanisms (i.e., lotteries over consumption goods and money) would only depend on the conditional expected money transfers. Hence we can indeed restrict ourselves w.l.o.g. to deterministic money transfers.

The ex ante expected utility of agent $i$ is

$$
\begin{aligned}
U_{\xi_{S}, \mu_{S}}^{i} & =\sum_{t^{i}} q\left(t^{i}\right) U_{\xi_{S}, \mu_{S}}^{i}\left(t^{i}\right) \\
& =\sum_{t} q(t)\left[\int_{\mathbb{R}_{+}^{l}} w^{i}\left(t, x^{i}\right) \xi_{S}\left(d x^{i} \mid t_{S}\right)+\mu_{S}^{i}\left(t_{S}\right)\right]
\end{aligned}
$$

The characteristic function of the exchange economy is then ${ }^{10}$ :

$$
V^{*}(S)=\left\{\begin{array}{l|l}
v \in \mathbb{R}^{n} \mid \begin{array}{c}
\exists \text { an incentive compatible mechanism } \\
\left(\xi_{S}, \mu_{S}\right) \text { for } S \text { such that } v^{i} \leq U_{\xi_{S}, \mu_{S}}^{i}
\end{array} \forall i \in S
\end{array}\right\}
$$

Transfers of ex ante utility can be carried out, e.g., through state independent monetary transfers, without affecting incentives. Thus $V^{*}$ can be simply described by the TU characteristic function

$$
v^{*}(S)=\max \left\{\sum_{i \in S} U_{\xi_{S}, \mu_{S}}^{i} \mid\left(\xi_{S}, \mu_{S}\right) \text { incentive compatible }\right\}
$$

According to the standard definition (see, e.g., Hildenbrand and Kirman (1988)), the core of $v^{*}$, denoted as $C\left(v^{*}\right)$, is the set of all payoff vectors $v \in \mathbb{R}^{N}$ which are feasible for the grand coalition (i.e., such that $\left.\sum_{i \in N} v^{i} \leq v^{*}(N)\right)$ and which cannot be blocked by any coalition $S$ (i.e., such that $\left.\forall S, \sum_{i \in S} v^{i} \geq v^{*}(S)\right)$. We refer to $C\left(v^{*}\right)$ (or to the corresponding set of random allocations) as the ex ante core of the economy.

For comparison, we also define the following characteristic functions:

$$
\begin{aligned}
v_{D}^{*}(S) & =\max \left\{\sum_{i \in S} U_{\xi_{S}, \mu_{S}}^{i} \mid \begin{array}{c}
\left(\xi_{S}, \mu_{S}\right) \text { deterministic } \\
\text { and incentive compatible }
\end{array}\right\} \\
v(S) & =\max _{\xi_{S}, \mu_{S}} \sum_{i \in S} U_{\xi_{S}, \mu_{S}}^{i} \\
v_{D}(S) & =\max \left\{\sum_{i \in S} U_{\xi_{S}, \mu_{S}}^{i} \mid\left(\xi_{S}, \mu_{S}\right) \text { deterministic }\right\}
\end{aligned}
$$

These characteristic functions are related as follows:

$$
v_{D}^{*}(S) \leq v^{*}(S) \leq v(S)=v_{D}(S), \text { for all } S .
$$

\footnotetext{
${ }^{10}$ Using, as in Holmström and Myerson (1983), "** for incentive compatible.
} 
The equality between $v(S)$ and $v_{D}(S)$ comes from the fact that random mechanisms cannot be useful in the absence of incentive compatibility constraints, because the utility functions $w^{i}(t,$.$) are concave. { }^{11}$ In the sequel, we shall not refer to $v_{D}$ anymore, but only to $v$. The presence of monetary transfers makes it possible to further simplify the characteristic function $v(S)$. It can be expressed in terms of the maximal aggregate utility from consumption goods, i.e., without reference to the monetary transfers, as follows: ${ }^{12}$

$$
v(S)=\max _{\xi_{S}}\left\{\sum_{i \in S} \sum_{t \in T} q(t) w^{i}\left(t, \xi_{S}^{i}\left(t_{S}\right)\right)\right\}
$$

the maximum being over all deterministic mechanisms $\xi_{S}$.

Remark 1. In general, (4) yields no logical inclusion between the various cores. However, if

$$
v_{D}^{*}(N)=v(N)
$$

we obviously have:

$$
C(v) \subseteq C\left(v^{*}\right) \subseteq C\left(v_{D}^{*}\right)
$$

By the complete information results (see, e.g., Hildenbrand and Kirman (1988)), $C(v)$ is not empty. Thus, it follows that all the ex ante cores are non-empty if (6) holds.

\section{GeneriC NON-EMPtiness}

In this section, we first state and prove our main positive result, that generically (in endowments) the full information first best is achievable.

We then identify specific assumptions which also guarantee the nonemptiness of the ex ante core (generically in the beliefs in proposition 1). For all these results, the basic idea will be to show that, thanks to monetary transfers and suitable assumptions, condition (6) holds. The non-emptiness of $C\left(v_{D}^{*}\right)$ and of $C\left(v^{*}\right)$ will then follow by remark 1 .

Theorem 1. If each agent's endowment is a one-to-one function of his type, $C\left(v^{*}\right)$ is non-empty. More precisely, $v_{D}^{*}=v$.

The idea is that incentive constraints are needed only to guard against claiming to be of a less wealthy type (in the vector sense) than one is in fact: types are partially verifiable.

\footnotetext{
${ }^{11}$ The first two inequalities in (4) can be strict - cf. footnote 7 .

${ }^{12} \mathrm{~A}$ similar simplification for $v^{*}(S)$ does not hold unless additional assumptions are made; see section 4 below.
} 
Proof. Fix a mechanism $\xi$ (i.e., without the transfers) for coalition $S$, which achieves $v(S)$. Let, for $t, a \in T^{i}, u_{t a}^{i}$ denote $i$ 's expected utility from $\xi$ when of type $t$ and claiming to be of type $a$, i.e., $u_{t a}^{i}=U_{\xi, 0}^{i}(t, a)$. Consider $\mu^{i} \in \mathbb{R}^{T^{i}}$ such that, whenever $e_{t}^{i} \geq e_{a}^{i}$ (i.e., $a$ is available to $t), \mu_{t}^{i}-\mu_{a}^{i} \geq u_{t a}^{i}-u_{t t}^{i}$ : clearly there exist such $\mu^{i}$ 's, by the one-to-one assumption: e.g., with $K=\max _{s, t}\left(u_{t s}^{i}-u_{t t}^{i}\right)$, let $\mu_{t}^{i}=K \cdot \#\left\{s \in T^{i} \mid e_{s}^{i} \leq e_{t}^{i}\right\} . \xi$ together with transfers $\mu_{t}^{i}$ to agent $i$ claiming to be of type $t$ is incentive compatible.

Whenever $(\xi, \mu)$ is incentive compatible, and, $\forall i \in N, \forall t \in T_{S}, \mu^{i}(t)$ depends only on $t^{i}$, then

$$
\nu^{i}(t)=\mu^{i}\left(t^{i}\right)-\frac{1}{\# S-1} \sum_{j \in S \backslash\{i\}} \mu^{j}\left(t^{j}\right)
$$

is such that $(\xi, \nu)$ is incentive compatible and budget-balanced.

In the previous proof, we constructed money transfers making first best allocations incentive compatible. This kind of construction is standard in the mechanism design literature (e.g., Arrow (1979), d'Aspremont and Gérard-Varet (1979, 1982), d'Aspremont, Crémer and Gérard-Varet (1990,1992), Crémer and McLean (1985), Green and Laffont (1979), Groves (1973), Groves and Loeb (1975), Johnson, Pratt and Zeckhauser (1990)). In the rest of the section, we shall use these results to establish some cases where the ex ante core is not empty.

Recall that the sum of the monetary transfers appears in the coalitions' objective function (see (1) and (3)). Large money transfers are likely to facilitate the fulfillment of the incentive compatibility constraints, but may also be costly. In the proof above as in some particular cases below, the argument is simplified by first ignoring the budget balancedness condition and relying on the following property:

Automatic Balance: For every incentive compatible mechanism $\left(\xi_{N}, \mu_{N}\right)$, there exists a money transfer scheme $\nu_{N}$ such that $\left(\xi_{N}, \nu_{N}\right)$ is incentive compatible and (budget balance):

$$
\sum_{i \in N} \nu_{N}^{i}(t)=0 \quad \forall t \in T
$$

d'Aspremont and Gérard-Varet $(1979,1982)$ identified assumptions (precisely stated below) on the prior $q$ which imply the previous property. A well-known, easy condition is that the agents' beliefs be independent, namely that $q(t)=\prod_{i \in N} q\left(t^{i}\right)$ (see Johnson, Pratt and Zeckhauser (1990)). Indeed, let $\left(\xi_{N}, \mu_{N}\right)$ be an incentive compatible mechanism. If the beliefs are independent, the mechanism $\left(\xi_{N}, \bar{\mu}_{N}\right)$ where

$$
\bar{\mu}_{N}^{i}(t)=\bar{\mu}_{N}^{i}\left(t^{i}\right)=\sum_{t^{-i}} q\left(t^{-i}\right) \mu_{N}^{i}\left(t^{i}, t^{-i}\right) \quad \forall i \in N, t \in T
$$


is incentive compatible. Then, as at the end of the above proof, there also exists $\nu$ s.t. $\left(\xi_{N}, \nu_{N}\right)$ is incentive compatible and budget-balanced.

Under automatic balance, the definition of $v^{*}(N)$ simplifies to:

$$
v^{*}(N)=\max _{\xi_{N}}\left\{\sum_{i \in N} \sum_{t \in T} q(t) \int_{\mathbb{R}_{+}^{l}} w^{i}\left(t, x^{i}\right) \xi_{N}\left(d x^{i} \mid t\right)\right\}
$$

where the maximum is over all mechanisms $\xi_{N}$ for which there exists a money transfer scheme $\mu_{N}$ such that $\left(\xi_{N}, \mu_{N}\right)$ is incentive compatible (the monetary transfers have disappeared from the objective function and the feasibility requirement on $\mu_{N}$ can be dispensed with). ${ }^{13}$

The first condition of d'Aspremont and Gérard-Varet $(1979,1982)$ on the prior $q$ can be formulated as follows ${ }^{14}$

Condition B. For every mapping $f: T \rightarrow \mathbb{R}$, there exists a money transfer scheme $\mu: T \rightarrow \mathbb{R}^{n}$ such that $\sum_{i \in N} \mu^{i}(t)=f(t)$ for every $t \in T$ and $\forall i \in N, \forall t^{i}, a^{i} \in T^{i}, a^{i} \neq t^{i}$

$$
\sum_{t^{-i}} q\left(t^{-i} \mid t^{i}\right) \mu^{i}\left(t^{i}, t^{-i}\right)>\sum_{t^{-i}} q\left(t^{-i} \mid t^{i}\right) \mu^{i}\left(a^{i}, t^{-i}\right)
$$

Clearly if the condition holds for $f=0$, it does for every $f$ (d'Aspremont, Crémer and Gérard-Varet $(1992,1995)$ ). The interpretation of condition B is straightforward: even if the agents' utility functions $w^{i}$ were identically zero (so that only the monetary transfers would generate utility), there would exist a balanced, strictly incentive compatible monetary transfer scheme. Hence, by multiplying these transfers by an appropriate constant, for any (typically not incentive compatible) mechanism $\xi_{N}$ on consumption goods, there exists a money transfer scheme $\nu_{N}$ satisfying $(7)$ such that $\left(\xi_{N}, \nu_{N}\right)$ is (strictly) incentive compatible. d'Aspremont, Crémer and Gérard-Varet (1992) also show that condition $\mathrm{B}$ is generic provided that $n \geq 3$ and no player is fully informed. Johnson, Pratt and Zeckhauser (1990) identify conditions on the agents' beliefs which are easy to check and imply condition B. In particular, letting $q^{i, i+1}(i+1$ mod. $n)$ denote agent $i$ 's conditional probability distribution (induced by $q$ ) over $T^{i+1}$, the following condition implies condition B:

$$
q^{i, i+1}\left(\cdot \mid t^{i}\right) \neq q^{i, i+1}\left(\cdot \mid s^{i}\right) \quad \forall i \in N, \forall t^{i}, s^{i} \in T^{i}, t^{i} \neq s^{i} .
$$

Proposition 1. Under condition $B, C\left(v^{*}\right)$ is non-empty. More precisely, (6) holds.

\footnotetext{
${ }^{13}$ This observation will also be useful in section 5 .

${ }^{14}$ As in d'Aspremont, Crémer and Gérard-Varet (1992), we use the "primal" form of the conditions initially introduced in d'Aspremont and Gérard-Varet (1979, 1982).
} 
Proof. If condition B holds, any deterministic mechanism $\xi_{N}$ which achieves $v(N)$ (see (5)) can be made incentive compatible thanks to a money transfer scheme $\nu_{N}$ satisfying (7). Hence, $v_{D}^{*}(N)$, given by (8), equals $v(N)$.

Remark 2. In fact, it suffices to assume that condition B holds just for those $a^{i}$ s.t. $e^{i}\left(a^{i}\right)=e^{i}\left(t^{i}\right)$. Indeed, one can then first add to the mechanism $\xi_{N}$ a sufficiently high multiple of the money transfer scheme $\mu$ of condition $\mathrm{B}$ (with $f=0$ ) to make sure that no deviations are profitable to another type with the same endowment. Add then to the mechanism a further transfer scheme, constructed as in theorem 1, to ensure that also deviations to a type with smaller endowment are not profitable.

Condition B is stronger than the next condition, obtained by weakening the inequalities:

Condition C. For every mapping $f: T \rightarrow \mathbb{R}$, there exists a money transfer scheme $\mu: T \rightarrow \mathbb{R}^{n}$ such that $\sum_{i \in N} \mu^{i}(t)=f(t) \forall t \in T$ and

$$
\sum_{t^{-i}} q\left(t^{-i} \mid t^{i}\right) \mu^{i}\left(t^{i}, t^{-i}\right) \geq \sum_{t^{-i}} q\left(t^{-i} \mid t^{i}\right) \mu^{i}\left(a^{i}, t^{-i}\right) \quad \forall i \in N, \forall t^{i}, a^{i} \in T^{i}
$$

The latter condition implies automatic balance (see Johnson, Pratt and Zeckhauser (1990)). Unlike in condition B, one cannot focus on $f=0$ here. Indeed, $\mathrm{C}$ would then hold for any beliefs (by taking $\mu=0$ ), which is not true (see d'Aspremont, Crémer and Gérard-Varet $(1992,1995))$.

As observed in d'Aspremont, Crémer and Gérard-Varet (1992, 1995), condition $\mathrm{C}$ is much weaker than B. E.g., independent beliefs cannot satisfy the latter (easy) but do satisfy the former (cf. d'Aspremont and Gérard-Varet $(1979,1982))$. In fact, d'Aspremont, Crémer and GérardVaret $(1992,1995)$ show that condition $\mathrm{B}$ is equivalent to $\mathrm{C}$ and the following condition of correlation between the agents' beliefs:

$$
q\left(\cdot \mid t^{i}\right) \neq q\left(\cdot \mid s^{i}\right) \quad \forall i \in N, \forall t^{i}, s^{i} \in T^{i}, t^{i} \neq s^{i}
$$

This property is called "belief announcement" in Johnson, Pratt and Zeckhauser (1990). The linear independence of agent $i$ 's beliefs $q\left(\cdot \mid t^{i}\right), t^{i} \in T^{i}$, which is much stronger than "belief announcement", is often considered in the literature (see e.g., Fudenberg and Tirole (1991), section 7.6). As pointed out above, Johnson, Pratt and Zeckhauser (1990) consider another strengthening of "beliefs announcement", by requiring the previous condition of correlation on the beliefs of every agent $i$ over agent $i+1$ (mod. $n$ ). 
In the next two propositions, we assume values are private, i.e.

$$
w^{i}\left(t, x^{i}\right)=w^{i}\left(t^{i}, x^{i}\right) \quad \text { for every } i \in N, t \in T \text { and } x^{i} \in \mathbb{R}_{+}^{l}
$$

Proposition 2. Under condition $C$ (in particular, if beliefs are independent), if values are private, then $C\left(v^{*}\right)$ is non-empty. More precisely, (6) holds.

Proof. As in the previous proof, take a mechanism $\xi_{N}$ which achieves $v(N): \xi_{N}$ is ex ante efficient. Make it ex post efficient by specifying:

$$
\xi_{N}(t) \in \arg \max _{x \in X_{N}(t)}\left\{\sum_{i \in N} w^{i}\left(t, x^{i}\right)\right\} \quad \text { if } q(t)=0
$$

By Groves (1973) (see also Green and Laffont (1979) and Groves and Loeb (1975)), there exists a money transfer scheme $\mu_{N}$ (not necessarily balanced) such that $\left(\xi_{N}, \mu_{N}\right)$ is incentive compatible. By condition C, $\mu_{N}$ can then be further made to satisfy automatic balance (argument stemming from d'Aspremont, Crémer and Gérard-Varet (1992)).

In the same vein, we deduce from d'Aspremont, Crémer and GérardVaret (1990):

Proposition 3. If $\# T^{i} \leq 2$ for every $i \in N$ and values are private, then $C\left(v^{*}\right)$ is non-empty. More precisely, $v_{D}^{*}=v$.

Proof. (d'Aspremont, Crémer and Gérard-Varet (1990)) show that $S$ has then an ex post efficient, incentive compatible mechanism $\left(\xi_{S}, \nu_{S}\right)$ satisfying (7). So, $v_{D}^{*}=v$.

The assumption $\# T^{i} \leq 2$ is very strong, but does not involve the beliefs $q$. It is not related with condition $\mathrm{C}$; for instance, if $n=2$, condition $\mathrm{C}$ is equivalent to independent beliefs (see d'Aspremont, Crémer and Gérard-Varet (1992)).

Remark 3. d'Aspremont, Crémer and Gérard-Varet (1990, 1992) point out that the mechanisms used in the proof of proposition 3 are incentive compatible in dominant strategies but are not Groves mechanisms. For an analysis of conditions under which Bayesian and dominant strategy incentive compatibility are equivalent, and the connection to Groves mechanisms, see Williams (1999). These conditions involve independent private values. Even in this particular case, the equivalence result in Williams (1999) does not apply to our model, since the set of types is finite.

Remark 4 . The positive results of this section typically rely on unlimited monetary transfers, and may not apply in the context of Walrasian economies in which the aggregate endowment of the transferable good 
is bounded. Indeed, we will show in section 6.2 that theorem 1 does not extend to Walrasian economies.

\section{An eXample in Which the EX ANTE CORE IS EMPTy}

In this section we show that with type-independent endowments, even in the quasi-linear setting, and even allowing for random mechanisms, non-emptiness of the ex ante core is not guaranteed. There are limits to how far the positive results can be pushed. We shall provide an example of an economy with quasi-linear utilities in which the ex ante incentive compatible core is empty. This will imply, in particular, that the assumption of endowments being a one-to-one function of types cannot be dropped from theorem 1, the assumption of private values cannot be dropped from proposition 2, and the assumption of separability cannot be dropped from the positive result in Forges and Minelli (1999). It will also show that the negative conclusion of Vohra (1999) is in fact completely robust as to the class of mechanisms considered as well as to additional desiderata for a "well behaved" economy. Interestingly, not only is our negative result stronger than that in Vohra (1999), the computations are also simpler: by the transferable utility setting, it suffices to show that the game is not balanced.

In the economy described below, $n=l=3$; agent 1 has two equiprobable types $s$ and $t$, while agents 2 and 3 have no private information $\left(T^{1}=\{s, t\}, q(s)=q(t)=\frac{1}{2}, T^{2}\right.$ and $T^{3}$ are singletons $)$.

Let the (state independent) endowments be

$$
e^{1}=(1,0,0), \quad e^{2}=(0,2,0), \quad e^{3}=(0,0,2)
$$

and the utility functions, with $x=\left(x_{1}, x_{2}, x_{3}\right)$ as consumption bundle:

$$
\begin{aligned}
w^{1}(s, x) & =2 x_{1}+f\left(x_{2}+x_{3}\right) \\
w^{2}(s, x)=w^{3}(s, x) & =3 x_{1}+g\left(x_{2}, x_{3}\right) \\
w^{1}(t, x) & =x_{1}+h\left(x_{2}\right)+h\left(x_{3}\right) \\
w^{2}(t, x)=w^{3}(t, x) & =x_{2}+x_{3}
\end{aligned}
$$

where

- $f: \mathbb{R}_{+} \rightarrow \mathbb{R}_{+}: x \mapsto \min \{x, 2\}$.

- $g: \mathbb{R}_{+}^{2} \rightarrow \mathbb{R}_{+}:(x, y) \mapsto 2 \min \{x, y\}$.

- $h: \mathbb{R}_{+} \rightarrow \mathbb{R}_{+}$is concave, with $h(0)=0, h(x) \leq x$, and $h \leq 1$.

For $C\left(v^{*}\right)$ to be empty, the positive result in Forges and Minelli (1999), theorem 1 , and proposition 2 (or 3) imply that the following three features are indispensable: (i) non-separability of the utility functions, given the structure of endowments, so of $g$, or non-linearity of 
$f$; (ii) the independence of endowments with respect to the state; (iii) the dependence of the utility functions of agents 2 and 3 on agent 1's type (non-private values).

Since $s$ and $t$ have probability $\frac{1}{2}$, we consider henceforth the sum of the utilities associated with each type, instead of the corresponding expected utility.

In order to show that $C\left(v^{*}\right)$ is empty, we follow the following steps:

Step 1: We compute $v$, and show that $v$ is exactly balanced, i.e.

$$
v(N)=\frac{1}{2}[v(\{1,2\})+v(\{1,3\})+v(\{2,3\})]
$$

Step 2: In subcoalitions, incentives entail no efficiency-loss:

$$
v_{D}^{*}(S)=v(S) \quad \text { for every } S:|S|=2
$$

Hence, by (4),

$$
v^{*}(S)=v(S) \text { for every } S:|S|=2
$$

Step 3: For $C\left(v^{*}\right)$ to be non empty, $v^{*}$ must be balanced (BondarevaShapley), i.e., by $(9)$ and $(10), v^{*}(N)=v(N)$. We will show that $v^{*}(N)<v(N)$ (assuming $\left.h(1)>0.5\right)$.

Before proceeding to the proofs of these three steps it will be useful to simplify the incentive constraints in this example.

Let $S$ be a coalition containing agent 1 . The beliefs of its members being trivially independent, $v^{*}(S)$ takes a simple form, as $v^{*}(N)$ in (8): (11)

$$
v^{*}(S)=\max _{\xi_{S}}\left\{\sum_{i \in S}\left[\int_{\mathbb{R}_{+}^{l}} w^{i}\left(s, x^{i}\right) \xi_{S}\left(d x^{i} \mid s\right)+\int_{\mathbb{R}_{+}^{l}} w^{i}\left(t, x^{i}\right) \xi_{S}\left(d x^{i} \mid t\right)\right]\right\}
$$

where the maximum is over all mechanisms $\xi_{S}$ which can be made incentive compatible for agent 1 by appropriate monetary transfers, i.e., for which there exist $\mu^{1}(s)$ and $\mu^{1}(t)$ such that

$$
\begin{gathered}
\int_{\mathbb{R}_{+}^{l}} w^{1}\left(s, x^{1}\right) \xi_{S}\left(d x^{1} \mid s\right)+\mu^{1}(s) \geq \int_{\mathbb{R}_{+}^{l}} w^{1}\left(s, x^{1}\right) \xi_{S}\left(d x^{1} \mid t\right)+\mu^{1}(t) \\
\int_{\mathbb{R}_{+}^{l}} w^{1}\left(t, x^{1}\right) \xi_{S}\left(d x^{1} \mid t\right)+\mu^{1}(t) \geq \int_{\mathbb{R}_{+}^{l}} w^{1}\left(t, x^{1}\right) \xi_{S}\left(d x^{1} \mid s\right)+\mu^{1}(s)
\end{gathered}
$$


i.e., for which ${ }^{15}$

$$
\begin{aligned}
& \int_{\mathbb{R}_{+}^{l}} w^{1}\left(s, x^{1}\right) \xi_{S}\left(d x^{1} \mid s\right)+\int_{\mathbb{R}_{+}^{l}} w^{1}\left(t, x^{1}\right) \xi_{S}\left(d x^{1} \mid t\right) \\
\geq & \int_{\mathbb{R}_{+}^{l}} w^{1}\left(s, x^{1}\right) \xi_{S}\left(d x^{1} \mid t\right)+\int_{\mathbb{R}_{+}^{l}} w^{1}\left(t, x^{1}\right) \xi_{S}\left(d x^{1} \mid s\right)
\end{aligned}
$$

So, as expected, monetary transfers play a double role here: they lead to a TU cooperative game, as under complete information, but they also facilitate the fulfillment of the incentive constraints.

5.1. Step 1. Without incentive constraints, efficiency of a deterministic mechanism, $x$, in the grand coalition requires, in state $s, x_{1}^{1}=0$, $x_{2}^{1}+x_{3}^{1} \leq 2, x_{2}^{i}=x_{3}^{i} \forall i$, leading to a total utility of 7 , and no trade with 1 in state $t$, hence total utility 5 . So,

$$
v(N)=12
$$

Efficiency in coalition $\{1,2\}$ (or $\{1,3\}$ ) similarly requires in state $s$ that both traders swap their endowments, leading to utility 5 , and no trade in state $t$, hence utility 3 . Thus:

$$
v(\{1,2\})=v(\{1,3\})=8
$$

And clearly, by trading to $(0,1,1),(0,1,1)$

$$
v(\{2,3\})=8 \text { also. }
$$

Thus the exact balancedness condition (9) holds.

5.2. Step 2. Coalition $\{2,3\}$ does not face any incentive compatibility constraint, so $v^{*}(\{2,3\})=v(\{2,3\})$.

Let us check that the first best allocation of coalition $\{1,2\}$ satisfies the incentive compatibility condition (14), namely that

$$
w^{1}(s,(0,2,0))+w^{1}(t,(1,0,0)) \geq w^{1}(s,(1,0,0))+w^{1}(t,(0,2,0))
$$

i.e., $2+1 \geq 2+h(2)$ : obvious. We have thus established (10).

5.3. Step 3. We want to prove that $v^{*}(N)<v(N)$. Suppose not. Then there is a first-best incentive compatible mechanism, i.e., satisfying (14) and carried by Eff $\times E_{s} f_{t}$, where Eff $f_{s}$ and Eff. denote the set of optimal allocations in states $s$ and $t$ respectively. But (14), when bringing all terms to one side, means there exists a probability distribution on $E f f_{s} \times E f f_{t}$ such that the expectation of some function is non-negative: i.e., this function is non-negative at some point. Thus, (14) is satisfied at some point in $E f f_{s} \times E f f_{t}$. Since further only player 1 's coordinates appear in (14), denote by Eff ${ }_{s}^{1}$ and $E f f_{t}^{1}$ the projections

\footnotetext{
${ }^{15}$ Lemma 3 in the appendix shows this in general.
} 
of $E f f_{s}$ and $E f f_{t}$ on player 1's coordinates, i.e. the set of commodity bundles to player 1 which are compatible in the corresponding state with a first best allocation.

We shall prove that (14) is violated everywhere on $\operatorname{Eff}_{s}^{1} \times \operatorname{Eff}_{t}^{1}$. Since, by step $1, \operatorname{Eff}_{s}^{1}=\{(0, x, x) \mid 0 \leq x \leq 1\}$ and $\operatorname{Eff}_{t}^{1}=\{(1,0,0)\}$, we get, using $u_{s}(\cdot)$ for the utility of player 1 of type $s$ as a function of his announcement ".", and similarly for $u_{t}$ :

$$
u_{s}(s)=2 x ; \quad u_{s}(t)=2 ; \quad u_{t}(s)=2 h(x) ; \quad u_{t}(t)=1
$$

The incentive constraints (14) become then:

$$
1+2 x \geq 2+2 h(x)
$$

which cannot be, since $2+2 h(x)-(1+2 x)$ is concave, and positive both at 0 and 1 (assuming $h(1)>0.5$ ).

5.4. Robustness of the example. Let preferences, endowments and the probability over the states of nature vary in the above framework (while keeping the basic structure: $n=l=3$; agent 1 has two types $s$ and $t$, agents 2 and 3 have no private information). Let us show that the ex ante core is empty in a neighborhood of the example: else, by proposition 4 in the appendix, there would be a sequence of economies with non-empty ex ante core converging to the example. Fix a mechanism in each of those cores. By proposition 5 (see appendix), one can extract a subsequence such that those mechanisms converge, and the limit is then an incentive compatible mechanism for our example, with expected utilities going to the limit. So some subcoalition can get more with an incentive compatible mechanism of its own, else any Pareto-efficient point in $V^{*}(N)$ that Pareto dominates this expected utility vector would belong to the core. So that subcoalition can get more with incentive compatible mechanisms in the limit than along the sequence. So it must be coalition $\{1,2\}$ (or $\{1,3\}$ ), since those are the only ones where incentive constraints matter. But step 2 showed that the incentive constraint for the first best mechanism of $\{1,2\}$ was satisfied strictly (assuming $h(2)<1$ ), hence it must still be satisfied in the neighborhood: contradiction.

In other words, for our example, there exists $\varepsilon>0$ and a finite set of consumption bundles $x_{\alpha}$ such that, whenever the probability on states, the endowments, and the utilities of each $x_{\alpha}$ are $\varepsilon$-close to those in the example, the ex ante core is still empty. So the example is completely robust as to additional requirements for a well-behaved economy (strictly positive endowments, utilities that are, in a neighborhood of the positive orthant, bounded and smooth with negative definite Hessian, etc.). 


\section{WALRASian ECONOMIES}

6.1. Robustness in a Stronger Sense. The characteristic function $v^{*}$ defined in section 3 by (3) is appropriate if the agents are allowed to make unlimited money transfers. We show now that the previous example can be used to construct a standard NTU economy, in which the agents' holdings of money must remain non-negative, and whose ex ante incentive compatible core is empty. We also show that robustness holds in an even stronger sense in this context.

For each subcoalition $S$, and every member of it, fix an efficient incentive compatible mechanism of $S$ giving this member the whole surplus above the individually rational levels. Each of those finitely many mechanisms involves, $T$ being finite, a finite number of possible money transfers away from each individual. Take the largest such amount (over all the above mechanisms, all individuals, and all $t \in T$ ), and give this to each trader as his initial endowment of money. This does not change anything. It just amounts to a translation of each player's utility scale. Now we can restrict money holdings to remain non-negative: the characteristic function $V^{*}$ (cf. (2)) can only decrease by this additional restriction; before the restriction it gave each coalition $S$ exactly the set of all utility vectors (to $S$ ) with sum not higher than $v^{*}(S)$; after the restriction, our specification of the initial endowments of money ensures that the extreme points of the individually rational part of the former $V^{*}(S)$ still belong to the new $V^{*}(S)$ : since this is convex, it follows that the individually rational part of $V^{*}$ did not change. Since the core depends only on this individually rational part, the core is unchanged too.

Recall that in section 5.4 we showed robustness with respect to the parameters of the economy, keeping fixed the set of states $\{s, t\}$. In the NTU case we will show that robustness holds in a stronger sense: one can allow for an arbitrary (finite) set of states, and information partitions, as long as the limit probability distribution is carried by two states which player 1 distinguishes and the others not.

Indeed, in the proof in section 5.4, upper semi-continuity in the TU case required eliminating transfers from the system in order to get a compact valued correspondence. In the NTU case, we get compactness for free since all mechanisms are carried by a fixed cube delimited by some upper bound on the aggregate endowments. So the correspondence to incentive compatible mechanisms is upper semi-continuous, since the incentive inequalities are weak.

We observed above that "the incentive constraint for the first best mechanism of coalition $\{1,2\}$ is satisfied strictly". This is the single TU incentive constraint, after eliminating transfers. But this implies 
that the set of transfers that solve the system of two incentive constraints is open. Thus some first best mechanism (including transfers) satisfies both incentive constraints strictly.

The rest of the proof is the same as in the TU case.

6.2. Type Dependent Endowments. The positive results of section 4 relied on the availability of unlimited monetary transfers. In particular, the proof of theorem 1 relied on a transfer mechanism giving bonuses to the wealthy types, and conversely for the less wealthy. In a Walrasian economy, such schemes may become infeasible, the possible transfers being bounded. And indeed, the core is empty in the following variation on our previous example:

Re-interpret it as Walrasian, i.e., without money, and where now

(i) Agent 1 has a higher endowment in state $t, e^{1}(t)=(1+\delta, 0,0)$; thereby satisfying the main hypothesis of theorem 1 .

(ii) The function $h$ has the form $h(x)=.5 \mathrm{~min}(x, 1)$.

For a pay-off vector $\vec{v}=\left(v^{1}, v^{2}, v^{3}\right)$, let $u=v^{1}-\delta, v=v^{2}+v^{3}$. Call $\vec{v}$ unblocked if no proper sub-coalition blocks it (with strict improvement for each coalition member, i.e., as in the weak core), and feasible if it is the expected utility vector of an incentive compatible mechanism.

Step 1. For $\vec{v}$ unblocked, $v \geq 8$.

Proof. If $v^{2}<2$ or $v^{3}<2$, agent 2 or 3 blocks on his own. Else coalition $\{2,3\}$ blocks by trading independently of the state to $(0, x, x),(0,2-$ $x, 2-x)$ for appropriate $x \in[0,2]-$ e.g., $x=1+\frac{v^{2}-v^{3}}{8}$.

Step 2. For $\vec{v}$ unblocked, $3 u+v \geq 19$.

Proof. If $u \geq 4$, this follows from step 1 . If $u<3$, agent 1 blocks on his own. And for $3 \leq u<4$, let agents 1 and 2 together use the incentive compatible mechanism that yields no-trade in state $t$, and in state $s$ the allocation $(x, 2),(1-x, 0)$ for $x=\frac{1}{2}(u-3+\varepsilon) \leq \frac{1}{2}$ : they get $v^{1}=u+\varepsilon+\delta, 2 v^{2}=19-3 u-3 \varepsilon$, so one must have $3 u+2 u^{2} \geq 19$, and similarly $3 u+2 u^{3} \geq 19$, hence the result.

Step 3. For $\vec{v}$ feasible, $5 u+3 v \leq 42$.

Proof. The mechanism is a pair of probability measures $\xi_{s}, \xi_{t}$ on allocations, with $v^{i}=\int w_{s}^{i}\left(x_{1}^{i}, x_{2}^{i}, x_{3}^{i}\right) d \xi_{s}+\int w_{t}^{i}\left(x_{1}^{i}, x_{2}^{i}, x_{3}^{i}\right) d \xi_{t}$.

The incentive constraint is thus:

$$
\int\left[x_{1}^{1}+.5 \min \left(x_{2}^{1}, 1\right)+.5 \min \left(x_{3}^{1}, 1\right)\right]\left[d \xi_{t}-d \xi_{s}\right] \geq \delta
$$


and the objective function $5 u+3 v$ equals:

$$
\begin{aligned}
& \int\left[10 x_{1}^{1}+9\left(x_{1}^{2}+x_{1}^{3}\right)+5 \min \left(x_{2}^{1}+x_{3}^{1}, 2\right)+6 \min \left(x_{2}^{2}, x_{3}^{2}\right)+6 \min \left(x_{2}^{3}, x_{3}^{3}\right)\right] d \xi_{s} \\
+ & \int\left[5\left(x_{1}^{1}-\delta\right)+2.5 \min \left(x_{2}^{1}, 1\right)+2.5 \min \left(x_{3}^{1}, 1\right)+3\left(x_{2}^{2}+x_{2}^{3}+x_{3}^{2}+x_{3}^{3}\right)\right] d \xi_{t}
\end{aligned}
$$

Thus it suffices to prove that their sum does not exceed 42, i.e. that:

$$
\begin{gathered}
\int\left[9\left(x_{1}^{1}+x_{1}^{2}+x_{1}^{3}\right)+5 \min \left(x_{2}^{1}+x_{3}^{1}, 2\right)+6 \min \left(x_{2}^{2}, x_{3}^{2}\right)+6 \min \left(x_{2}^{3}, x_{3}^{3}\right)\right. \\
\left.-.5 \min \left(x_{2}^{1}, 1\right)-.5 \min \left(x_{3}^{1}, 1\right)\right] d \xi_{s} \\
+3 \int\left[2 x_{1}^{1}+\min \left(x_{2}^{1}, 1\right)+x_{2}^{2}+x_{2}^{3}+\min \left(x_{3}^{1}, 1\right)+x_{3}^{2}+x_{3}^{3}\right] d \xi_{t}-6 \delta \leq 42
\end{gathered}
$$

The second integrand is $\leq 6+2 \delta$, since $x_{1}^{1} \leq 1+\delta, \sum x_{2}^{i} \leq 2, \sum x_{3}^{i} \leq 2$. There remains thus to show that the first is no more than 24 .

Since $x_{2}^{1}+\min \left(x_{2}^{2}, x_{3}^{2}\right)+\min \left(x_{2}^{3}, x_{3}^{3}\right) \leq 2$, and similarly for $x_{3}^{1}$, it follows that $\min \left(x_{2}^{2}, x_{3}^{2}\right)+\min \left(x_{2}^{3}, x_{3}^{3}\right) \leq 2-\max \left(x_{2}^{1}, x_{3}^{1}\right)$. Thus, since also $\sum x_{1}^{i} \leq 1$, the first integrand is majorised by:

$$
21+5 \min \left(x_{2}^{1}+x_{3}^{1}, 2\right)-6 \max \left(x_{2}^{1}, x_{3}^{1}\right)-.5 \min \left(x_{2}^{1}, 1\right)-.5 \min \left(x_{3}^{1}, 1\right) .
$$

This increases when decreasing the larger of $x_{2}^{1}$ and $x_{3}^{1}$. So it is, for $y=$ $\min \left(x_{2}^{1}, x_{3}^{1}\right)$, majorised by $21+5 \min (2 y, 2)-6 y-\min (y, 1) \leq 24$.

Step 4. The weak core is empty.

Proof. 3 times the inequality of step 3 minus 5 times that of step 2 yields $4 v \leq 31$, contradicting step 1 .

\section{The Interim Stage}

It is natural to ask whether our results extend to a similar notion of core for the case where decisions are made at the interim stage.

Wilson (1978) provided a seminal analysis of the core at the interim stage (without imposing incentive constraints). An important concept developed in Wilson (1978) was that of the coarse core, in which coalitions coordinate on mechanisms at the interim stage and rely on a common knowledge informational event to evaluate their interim utilities. Wilson proved that the coarse core is non-empty under standard conditions. ${ }^{16}$ However, Vohra (1999) showed that Wilson's positive

\footnotetext{
${ }^{16}$ The notion of the fine core in Wilson (1978) is not relevant for our purposes since, as shown in Wilson (1978), it can be empty even without the imposition of incentive constraints.
} 
result does not extend to the case in which incentive constraints are imposed, i.e., the incentive compatible coarse core may be empty.

Is it possible to obtain non-emptiness of the incentive compatible coarse core by permitting monetary transfers? Unfortunately, the approach of section 4, which was so fruitful in the ex ante case, does not immediately extend to the interim case. While monetary transfers allow us to transfer ex ante utility across consumers without affecting incentive constraints, the same need not be true in terms of transfers of interim utility (across types). Indeed, one ingredient of the approach in section 4 was to construct, corresponding to an efficient outcome, a transfer scheme satisfying incentive compatibility. And an appropriate transfer scheme will typically affect interim utilities. Indeed, restrictions on interim utility (such as interim individual rationality) may be too demanding if one insists on first-best efficiency. It is possible, even in an economy with independent, private values that there exists no incentive compatible mechanism which is both interim individually rational and first best efficient; see, for example, Table 5.2 in Milgrom and Roberts (1992). ${ }^{17}$ Thus the kind of argument used in section 4 to obtain positive results cannot be extended to the incentive compatible coarse core.

Recall that our approach in section 4 relied on showing that, under suitable assumptions, incentive constraints did not preclude first-best efficiency. Non-emptiness then followed easily from remark 1 . While that approach may no longer be fruitful, the possibility remains open that the incentive compatible coarse core is non-empty if conditions such as $\mathrm{B}$ or $\mathrm{C}$ hold. ${ }^{18}$ In particular, it is not known to us whether the incentive compatible coarse core is generally non-empty in the case of independent private values or the case in which the endowment of each agent is a one-to-one function of his type. These are important questions that still remain open.

However, we can show that the incentive compatible coarse core is empty in the example of section 5, thereby strengthening the conclu-

\footnotetext{
${ }^{17}$ The only reason we cannot directly apply the impossibility result of Myerson and Satterthwaite (1983) here is that they require a continuum of types.

${ }^{18}$ An example such as that of Milgrom and Roberts (1992) we mentioned above cannot be invoked to settle this issue negatively. That result concerns first-best efficiency; allocations in the incentive compatible coarse core are only required to be incentive efficient. Indeed, in a two-agent economy (such as the one considered in Milgrom and Roberts (1992) or Myerson and Satterthwaite (1983)), the incentive compatible coarse core corresponds to the set of interim incentive efficient and interim individually rational mechanisms, and is, therefore, non-empty under standard assumptions.
} 
sion in Vohra (1999); quasi-linearity and randomness are not enough to guarantee the non-emptiness of the incentive compatible coarse core.

In our example, the incentive compatible coarse core ${ }^{19}$ differs from the ex ante incentive compatible core for precisely the following reasons. A coarse objection for a coalition containing agent 1 and some uninformed consumer(s) requires increasing the ex ante utility of each uninformed consumer and increasing the utility of consumer 1 in both states. Coalition $\{1\}$ has a coarse objection if consumer 1 can obtain a higher utility in either state with his own endowments. (A coarse objection by a coalition which does not contain agent 1 is exactly the same as an ex ante objection).

To refine our earlier conclusion that $v^{*}(N)<12$, we first show that

$$
v^{*}(N) \leq 13-2 h(1) \text {. }
$$

Incentive compatibility (IC) of the mechanism $\xi(s), \xi(t)$ means that:

$$
\int\left(w^{1}\left(s, x^{1}\right)-w^{1}\left(t, x^{1}\right)\right)\left(\xi\left(d x^{1} \mid s\right)-\xi\left(d x^{1} \mid t\right)\right) \geq 0
$$

and then our objective function (OF) should satisfy:

$$
\mathrm{E}_{\xi(s)} \sum_{1}^{3} w^{i}\left(s, x^{i}\right)+\mathrm{E}_{\xi(t)} \sum_{1}^{3} w^{i}\left(t, x^{i}\right) \leq 13-2 h(1)
$$

Consider first the allocation to agents 2 and 3, who don't appear in IC. By concavity of $w^{2}\left(s, x^{2}\right)+w^{3}\left(s, x^{3}\right)$ and $w^{2}\left(t, x^{2}\right)+w^{3}\left(t, x^{3}\right)$, it is better to replace $\left(x^{2}, x^{3}\right)$ by its conditional expectation given $x^{1}$. Assume, by monotonicity, that they share everything agent 1 does not get (i.e., no free-disposal); and, since $w^{2}=w^{3}$, that they share it equally: hence, $x^{2}=x^{3}=\frac{1}{2}\left[(1,2,2)-x^{1}\right]$, and we can now view $\xi(s), \xi(t)$ as probability distributions over $Y=\left\{x \in \mathbb{R}_{+}^{3} \mid x_{1} \leq 1, x_{2}, x_{3} \leq 2\right\}$.

By additive separability and linearity of $x_{1}$ in all utility functions, this coordinate can be replaced in the mechanism by its expectation, without affecting either IC or OF. Thus we get $x_{s}, x_{t} \in[0,1]$, and $\xi(s), \xi(t)$ are distributions on $[0,2]^{2}$. To lighten notation, we will henceforth use $x, y, z$ for $x_{1}, x_{2}, x_{3}$. So we can rewrite our claim:

$$
\begin{aligned}
& \int[f(y+z)-h(y)-h(z)][\xi(d y, d z \mid s)-\xi(d y, d z \mid t)] \geq x_{t}-x_{s} \Longrightarrow \\
& 2 x_{s}+3\left(1-x_{s}\right)+\int\left[f(y+z)+2 g\left(1-\frac{y}{2}, 1-\frac{z}{2}\right)\right] \xi(d y, d z \mid s) \\
& \quad+x_{t}+\int[h(y)+h(z)-y-z] \xi(d y, d z \mid t)+4 \leq 13-2 h(1)
\end{aligned}
$$

\footnotetext{
${ }^{19}$ See Vohra (1999) for a general definition.
} 
So, eliminating $x_{t}-x_{s}$, it suffices to prove that:

$$
\begin{gathered}
\int[f(y+z)-h(y)-h(z)][\xi(d y, d z \mid s)-\xi(d y, d z \mid t)]+ \\
\int\left[f(y+z)+2 g\left(1-\frac{y}{2}, 1-\frac{z}{2}\right)\right] \xi(d y, d z \mid t)+ \\
\int[h(y)+h(z)-y-z] \xi(d y, d z \mid t) \leq 6-2 h(1)
\end{gathered}
$$

It suffices therefore to prove that, on $[0,2]^{2}$ :

from $\xi(s): 2 f(y+z)+2 g\left(1-\frac{y}{2}, 1-\frac{z}{2}\right)-h(y)-h(z) \leq 6-2 h(1)$

from $\xi(t): \quad 2 h(y)-y+2 h(z)-z-f(y+z) \leq 0$

For the second inequality, best is to equalize $y$ and $z$, at $y+z$ constant, by concavity of $h$. So, replacing $f$, it amounts to $2 h(x)-x-\min (x, 1) \leq$ 0 , which follows from $h(x) \leq \min (x, 1)$.

Replacing now $f$ and $g$ in the first, there remains to prove:

$$
2 \min (y+z, 2)-2 \max (y, z)-h(y)-h(z) \leq 2-2 h(1)
$$

To increase the left-hand member, one should clearly decrease $y$ and $z$ if $y+z>2$. Thus $y+z \leq 2$, and hence the objective equals $2 \min (y, z)-h(y)-h(z)$ : one should decrease the maximum of $y$ and $z$. We get then $y=z \leq 1$, where the objective $2 y-2 h(y)$ is increasing: the maximum is at $y=z=1$, and yields equality. This completes the proof that $v^{*}(N) \leq 13-2 h(1)$.

Now assume that $h(1)>.75$, so that $v^{*}(N)<11.5$. Suppose there exists a mechanism, $(\xi, \mu)$, which belongs to the incentive compatible coarse core. Corresponding to this mechanism, let $u^{i}(\omega)$ refer to the utility of consumer $i$ in state $\omega$ and $U^{i}$ the sum of utilities of consumer $i$ in the two states. Let the sum of utilities of the three consumers in both states be denoted $U=u^{1}(s)+u^{1}(t)+U^{2}+U^{3}$.

We know that if $U^{2}+U^{3}<8$, then coalition $\{2,3\}$ has an ex-ante incentive compatible objection. Since this is a coalition of uninformed agents, this is also a coarse objection, which contradicts the hypothesis that this mechanism is in the incentive compatible coarse core. Thus we may henceforth assume that $U^{2}+U^{3} \geq 8$.

Interim individual rationality implies that $u^{1}(s) \geq 2$ and $u^{1}(t) \geq 1$. This along with the condition $U^{2}+U^{3} \geq 8$ implies that there exist $\delta(s), \delta(t)$ and $\delta$, all non-negative, such that

$u^{1}(s)=2+\delta(s), u^{1}(t)=1+\delta(t), U^{2}+U^{3}=8+\delta, \delta(s)+\delta(t)+\delta<0.5$.

Without loss of generality, $U^{2} \leq 4+0.5 \delta$. Consider coalition $\{1,2\}$ and the incentive compatible mechanism in which they swap their goods in 
state $s$ and there is no trade in state $t$. This yields utilities,

$$
w^{1}(s)=2, w^{1}(t)=1, w^{2}(s)=3, w^{2}(t)=2 .
$$

Suppose consumer 2 transfers to consumer 1 , in each state (thereby retaining incentive compatibility) the amount $m=[\delta(s)+\delta(t)] / 2+1 / 4$. Since $\delta(s)<0.5$ and $\delta(t)<0.5, m>\max (\delta(s), \delta(t))$. This means that consumer 1 is better-off in both states. Consumer 2 is also better off, receiving the sum of utilities

$$
5-[\delta(s)+\delta(t)+1 / 2]>5-[1-\delta] \geq 4+0.5 \delta .
$$

Thus $\{1,2\}$ has an incentive compatible coarse objection; contradiction.

THEMA, Université de Cergy-Pontoise, 33, Boulevard du Port 95011, Cergy-Pontoise Cedex, France; Francoise.Forges@eco.u-cergy.fr

CORE, Université Catholique de Louvain, 34, Voie du Roman-Pays, B-1348Louvain-la-Neuve, Belgique; jfm@core.ucl.ac.be

$$
\text { and }
$$

Dept. of Economics, Brown University, Providence, R.I. 02912, U.S.A.; Rajiv_Vohra@brown.edu; http://www.econ.brown.edu/〜rvohra 


\section{APPENDIX: Continuity of The InCEntive COMPATIBLE MECHANISMS AS A FUNCTION OF THE ECONOMY}

Lemma 1. Let $C$ be a finite-dimensional compact convex set, and, with $A$ a directed set and $\alpha \in A$, let $f, f_{\alpha}$ be concave on $C$, and $f$ real-valued and continuous. If $f_{\alpha}$ is uniformly bounded below and $\lim \sup f_{\alpha} \leq f$, then $\forall \varepsilon>0, \exists \alpha_{0} \in A: f_{\alpha} \leq f+\varepsilon \forall \alpha \geq \alpha_{0}$.

Proof. Since $f$ is, by Dini's theorem (and the separation theorem), the uniform limit of the minima of finitely many affine functions $\phi$ that lie everywhere strictly above it, it suffices to show that for any such $\phi$ there exists $\alpha_{0}$ s.t., for $\alpha \geq \alpha_{0}, f_{\alpha} \leq \phi$. Subtracting then $\phi$ from all functions, we have reduced the problem to the case where $f=-\varepsilon$. And assume w.l.o.g. that $f_{\alpha} \geq-1 \forall \alpha$, and that $C$ is full-dimensional.

Let $r$ denote the radius of the largest ball contained in $C$. For $x \in C$, denote by $f_{x}$ the smallest concave function on $C$ which is $\geq-1$ everywhere and $\geq 0$ at $x$. Note that $\left\{y \in C \mid f_{x}(y)>-\varepsilon / 2\right\}$ contains an open ball (centered at some point of $C$ ) of radius $r \varepsilon / 2$. Let then $F$ be a finite subset of $C$ that intersects every such ball (i.e., s.t. any point of $C$ is at distance less than $r \varepsilon / 2$ from some point in $F$ : e.g., the centers of a finite open covering by such balls). Choose then $\alpha_{0}$ s.t., $\forall \alpha \geq \alpha_{0}$, $f_{\alpha}(x)<\varepsilon / 2 \forall x \in F$.

Lemma 2. Let $C$ be a finite-dimensional compact convex polyhedron. If a net of concave functions on $C$ converges pointwise to a real-valued continuous function on $C$, it does so uniformly.

Proof. $f$ is concave as a limit of concave functions. Given a barycentric subdivision $s$ of the polyhedron, denote by $f_{s}$ the piece-wise linear function (on $s$ ) that coincides with $f$ at all vertices of $s: f \geq f_{s}$, and, by the uniform continuity of $f$, one can choose $s$ sufficiently fine that $f_{s}>f-\varepsilon$. Choose then $\alpha_{0}$ s.t., $\forall \alpha \geq \alpha_{0}, f_{\alpha}>f-\varepsilon$ at every vertex of $s-$ so, by concavity, $f_{\alpha}>f_{s}-\varepsilon>f-2 \varepsilon$ everywhere. The previous lemma gives now the other half of the conclusion.

Proposition 4. Let $U$ be the space of all concave continuous functions on $\mathbb{R}_{+}^{l}$ which are bounded below. Fix a probability $\mu$ on $\mathbb{R}_{+}$with finite first order moment and unbounded support, and, for $u, v \in U$, let $d(u, v)=\int_{0}^{\infty} \sup _{\|x\| \leq r}|u(x)-v(x)| \mu(d r)$. Then $(U, d)$ is a complete, separable metric space, and its topology, the pointwise topology.

Proof. Observe first that any $u \in U$ is monotone: $u(y)<u(x)$ for $y \geq x$ would by concavity imply that $\lim _{t \rightarrow \infty} u((1-t) x+t y)=-\infty$. Next, consider a pointwise convergent net $u(\alpha) \rightarrow u$ in $U$ : by the previous lemma, $u(\alpha) \rightarrow u$ uniformly on compact sets (each of them being 
contained in a compact, convex polyhedron in $\mathbb{R}_{+}^{l}$ ). So, for $\alpha \geq \alpha_{0}$, $\sup _{\|x\| \leq 1}\left|u_{\alpha}(x)-u(x)\right| \leq 1$ (using for $\|\cdot\|$ the $\ell_{1}$-norm). Let $M=$ $1+\sup _{\|x\| \leq 1}|u(x)|$ : then, for $\alpha \geq \alpha_{0}, u_{\alpha}(x) \geq-M \forall x$ by monotonicity, and $u_{\alpha}(x) \leq-M+2 M\|x\|$ for $\|x\| \geq 1$ by concavity, so $\left\|u_{\alpha}(x)\right\| \leq$ $M+2 M\|x\| \forall x$. This implies first that $d(u, 0)<\infty$, so $d(u, v)<$ $\infty \forall u, v \in U: d$ is a distance. Further, the same bound yields, by the previous lemma and the dominated convergence theorem (which applies here to nets, since $f_{\alpha}(r)=\sup _{\beta>\alpha} \sup _{\|x\| \leq r}\left|u_{\beta}(x)-u(x)\right|$ decreases to 0 uniformly on compact sets), that $d\left(u_{\alpha}, u\right) \rightarrow 0$ : the $d$-topology is weaker than the point-wise topology - and the converse is obvious. Given this, the minima of finitely many affine functions with rational coefficients are clearly dense: $(U, d)$ is separable; and the completeness is clear.

Remark 5. As $x \wedge n \rightarrow x$ shows, $\sup _{r}\left(1 \wedge r^{-1}\right) \sup _{\|x\| \leq r}|u(x)-v(x)|$ would not be an appropriate distance.

The next lemma is for the sake of completeness: Johnson, Pratt and Zeckhauser (1990) do this even without independence (cf. also Crémer and McLean (1985)).

Lemma 3. The projection on $\Delta\left(X_{N}\right)^{T}$ of the set of incentive compatible mechanisms for an economy with independent types is the set of solutions of the system: $\forall i \in N, \forall n>1, \forall\left(t_{k}^{i}\right)_{k \in \mathbb{Z}_{n}} \in\left(T^{i}\right)^{\mathbb{Z}_{n}}$

$$
\sum_{k \in \mathbb{Z}_{n}}\left(g_{i}\left(t_{k}^{i}, t_{k}^{i}\right)-g_{i}\left(t_{k}^{i}, t_{k+1}^{i}\right)\right) \geq 0
$$

where, for $s, t \in T^{i}, g_{i}(s, t)=\sum_{t^{-i}} q\left(t^{-i}\right) \int_{\mathbb{R}_{+}^{l}} w^{i}\left(s, t^{-i}, x^{i}\right) \xi\left(d x^{i} \mid t, t^{-i}\right)$.

Proof. Assume for notational simplicity that $\forall i, \forall t, w^{i}(t, 0)=0$. It suffices to express the system of incentive constraints with one transfer for each type of each agent (so: $\sum_{i \in N} \# T^{i}$ independent real variables), since one can then always afterwards balance the transfers as at the end of the proof of theorem 1. Eliminating by Farkas' lemma the transfers from this system yields

$$
\sum_{s, t} A_{s, t}\left[g_{i}(t, t)-g_{i}(t, s)\right] \geq 0
$$

where $A$ is a non-negative $T^{i} \times T^{i}$-matrix with $\forall t_{0} \in T^{i}, \sum_{t} A_{t, t_{0}}=$ $\sum_{t} A_{t_{0}, t}$, (and $A_{t_{0}, t_{0}}=0$ ). The extreme points of this set are clearly the cycles, i.e. those matrices $A$ where the $A_{s, t}$ as well as the row and column sums belong to $\{0,1\}$. Think indeed of $A_{s, t}$ as representing a flow from $s$ to $t$ : the conditions express that inflow equals outflow at each point. It is then clear how to decompose such a flow as a sum of cycles. 
Remark 6. Henceforth it is this projection that we will call the set of incentive compatible mechanisms.

Proposition 5. Topologize the space of economies with the usual topology on $\Delta(T)$ and on the endowments, and the point-wise topology on each $u_{t}^{i}$. With the weak topology on $\left(\Delta X_{N}\right)^{T}$, the correspondence from economies with independent types to incentive compatible mechanisms is compact, convex valued, and upper semi-continuous.

Proof. The convexity is clear from the linearity of inequalities (15). For the rest, we take a convergent sequence (by proposition 4) of such economies, together with an incentive compatible mechanism for each, and show that we can extract a subsequence along which the mechanisms converge too, and that then the limiting mechanism is an incentive compatible mechanism for the limiting economy. Since endowments converge, the set of all feasible allocations, for all those economies, is contained in some fixed big cube. Hence our sequence of mechanisms, as a sequence of probabilities on this cube, contains a weak ${ }^{*}$-convergent subsequence. Assume thus it is itself convergent. Continuity of the endowments ensures that the limiting distribution is feasible, a mechanism. And the fact that the integrals of a uniformly convergent sequence of continuous functions w.r.t. a weakly convergent sequence of probabilities go to the limit ensures that the (weak) inequalities (15), hence the incentive compatibility, remain true in the limit.

Corollary 1. $v^{*}$ is upper semi-continuous on the space of economies with independent types.

Proof. Since each coalition $S$ is such an economy on its own, this follows from proposition 5, the sum of expected utilities (cf. (8)) being a continuous function on the graph of that correspondence, by continuity of the integrals of a uniformly convergent sequence of continuous functions w.r.t. a weakly convergent sequence of probabilities.

Remark 7 . While the nature of the arguments up to here is needed even if one were just interested in proving the example robust (as seen in section 5.4), this is no longer true for those that follow, which are there just as a first attempt to elucidate the robustness in our framework of the concepts of characteristic function and of incentive compatibility.

In the sequel, we basically show that a variation on the argument used above and in section 5.4 works in general, i.e., if incentive constraints are not satisfied strictly, the mechanism can be slightly perturbed so as to satisfy them strictly. 
Lemma 4. The incentive compatible mechanisms of an economy with independent types are the solutions of system (15), where further, for $k, l \in \mathbb{Z}_{n}, k \neq l \Rightarrow t_{k}^{i} \nsim t_{l}^{i}$, defining, for $s, t \in T^{i}, s \sim t \Longleftrightarrow$ $\forall t^{-i} \in \prod_{j \neq i} T^{j}, \forall x \in \mathbb{R}_{+}^{l}: x \leq \sum_{j \in N} e^{j} \Rightarrow w^{i}\left(s, t^{-i}, x\right)-w^{i}\left(s, t^{-i}, 0\right)=$ $w^{i}\left(t, t^{-i}, x\right)-w^{i}\left(t, t^{-i}, 0\right)$.

Its interior is non-empty in the weak* space of all mechanisms, and all those inequalities are strict there.

Proof. Assume for notational simplicity that $\forall i, \forall t, w^{i}(t, 0)=0$. For the first part, take a cycle, as obtained from lemma 3, where, for some $k \neq l, t_{k} \sim t_{l}$. Numbering can start at one of them, so $1 \leq l<n$, and $t_{l} \sim t_{0}$. Then the sum corresponding to this cycle, numbered from 1 to $n$, decomposes into the two sums from 1 to $l$ and from $l+1$ to $n$, by the equalities $g\left(t_{n}, t_{1}\right)=g\left(t_{l}, t_{1}\right)$ and $g\left(t_{l}, t_{l+1}\right)=g\left(t_{n}, t_{l+1}\right)$, which follow directly from $t_{l} \sim t_{n}$. Thus the first part, a cycle of length 1 being an identity.

As to the second part, note first that, given a finite system of continuous linear weak inequalities on a compact, convex set in a locally convex space, either all of them are satisfied strictly at some point, so the solution set has non-empty interior, or some convex combination of them is never so, i.e., the corresponding equality holds. Indeed, just map everything to Euclidean space, using the given linear functionals: if the (compact, convex) image is disjoint from the interior of the positive orthant, they can be weakly separated by a non-zero linear functional; this must be non-negative, and normalizing it to 1 yields then a convex combination of the original functionals.

We apply this to our incentive constraints: assuming that there exists no mechanism where all inequalities are strict, we get on the (weak*) compact, convex set of all mechanisms:

$$
\sum_{i} \sum_{s, t \in T^{i} \times T^{i}} A_{s, t}^{i}\left[g_{i}(t, t)-g_{i}(t, s)\right] \leq 0
$$

for any mechanism $\xi$, where $A^{i}$ is a positive linear combination of cycle matrices as used in the first part.

Then, since any $\xi$ can be modified such as to distribute 0 to all $i \neq i_{0}$, the sum over $i$ can as well be limited to $i=i_{0}$; i.e., we can assume $A_{s, t}^{i}=0$ for $i \neq i_{0}$. So, each individual summand in the sum over $i \in I$ must be $\leq 0$, and for at least one of them the matrix $A^{i}$ contains a non-trivial cycle in the sense of the first part. There is then no loss of generality to assume this $i$ to be 1 , and $A^{i}=0$ for $i \neq 1$. Further, since only the coordinate $x^{1}$ of the allocation selected by $\xi$ appears in the system now, we can just think of $\xi$ as a distribution, indexed by $t \in T$, over $Y=\left\{x \in \mathbb{R}_{+}^{l} \mid x \leq \sum_{i} e^{i}\right\}$. 
Now, similarly, we can set to zero all coordinates of the allocation corresponding to $t^{-1} \neq t_{0}^{-1}$, for any fixed $t_{0}^{-1}$, so the inequality has to hold not only for the $g_{1}$, but for each $g_{t^{-1}}\left(t^{-1} \in \prod_{j \neq 1} T^{j}\right.$, with $\left.q\left(t^{-1}\right)>0\right)$, defined by $g_{t^{-1}}(s, t)=\int_{\mathbb{R}_{+}^{l}} w^{1}\left(s, t^{-1}, x\right) \xi\left(d x^{i} \mid t, t^{-1}\right)$ :

$$
\begin{aligned}
& \sum_{T^{1} \times T^{1}} A_{s, t}\left[g_{t^{-1}}(t, t)-g_{t^{-1}}(t, s)\right] \leq 0 \quad \forall \xi, \forall t^{-1} \in \prod_{j \neq 1} T^{j}: q\left(t^{-1}\right)>0, \text { i.e. } \\
& \sum_{T^{1} \times T^{1}} A_{s, t}\left[w^{1}\left(t, t^{-1}, x_{t}\right)-w^{1}\left(t, t^{-1}, x_{s}\right)\right] \leq 0 \quad \forall x \in Y^{T^{1}}, \forall t^{-1}: q\left(t^{-1}\right)>0
\end{aligned}
$$

Since $x$ varies in a product $Y^{T^{1}}$, and the sum decomposes into terms depending each only on a single coordinate of $x$, and vanishing with it, it must be that each partial sum over terms implying only a given coordinate of $x$ is already $\leq 0: \forall t \in T^{1}, \forall x\left(=x_{t}\right) \in Y, \forall t^{-1}: q\left(t^{-1}\right)>0$ :

$$
\sum_{s \in T^{1}} A_{s, t} w^{1}\left(t, t^{-1}, x\right) \leq \sum_{s \in T^{1}} A_{t, s} w^{1}\left(s, t^{-1}, x\right)
$$

Let $T^{-1}=\prod_{j \neq 1} T_{0}^{j}$, where $T_{0}^{j}=\left\{t \in T^{j} \mid q(t)>0\right\}$, and, $\forall t \in T^{1}, v_{t}: T^{-1} \times Y \rightarrow \mathbb{R}:\left(t^{-1}, x\right) \mapsto w^{1}\left(t, t^{-1}, x\right)$. Then, IC conditions being needed only on $T_{0}^{1}$, so cycles are carried by $T_{0}^{1}$ (hence $A_{s, t}=0$ outside $T_{0}^{1} \times T_{0}^{1}$ ), the above means

$$
\sum_{s \in T_{0}^{1}} A_{s, t} v_{t} \leq \sum_{s \in T_{0}^{1}} A_{t, s} v_{s}
$$

I.e., let $\forall t \in T_{0}^{1}, \sigma_{t}=\sum_{s \in T_{0}^{1}} A_{s, t}=\sum_{s \in T_{0}^{1}} A_{t, s}$, and $T^{\prime}=\left\{t \mid \sigma_{t}>0\right\}$ :

$$
\sigma_{t} v_{t} \leq \sum_{s \in T^{\prime}} A_{t, s} v_{s}, \text { where } \sigma_{t}=\sum_{s \in T^{\prime}} A_{t, s}>0
$$

Let $\epsilon_{t}\left(t^{-1}, x\right) \geq 0$ be the corresponding slack: by summing over $t \in T^{\prime}$, $\sum_{t} \sigma_{t} v_{t}+\sum_{t} \epsilon_{t}=\sum_{s, t} A_{t, s} v_{s}=\sum_{t} \sigma_{t} v_{t}$, hence $\forall t \in T^{\prime}, \epsilon_{t}=0$. So:

$$
v_{t}=\frac{1}{\sigma_{t}} \sum_{s \in T^{\prime}} A_{t, s} v_{s} \quad \forall t \in T^{\prime}
$$

$A$ being a sum of non-trivial cycles means $t \nsim s$ if $A_{t, s}>0$, i.e., $v_{t} \neq v_{s}$ : each $v_{t}$ for $t \in T^{\prime}$ is a convex combination of the others: contradiction, for $v_{t}$ extreme in the convex hull of $\left\{v_{s} \mid s \in T^{\prime}\right\}$.

Proposition 6. The correspondence of proposition 5 is continuous on the open, dense set of economies where any two different types of any agent have, for at least some vector of types of the others, different preferences over the feasible set. ("preference announcement") 
Proof. Openness and density are clear. Upper semi-continuity was obtained in proposition 5. For the lower semi-continuity, the $\sim$ equivalence classes being singletons at those economies, any incentive constraint that occurs in the neighborhood is, by lemma 4 , satisfied strictly at an interior point, which is thus still interior in the neighborhood.

Corollary 2. $v^{*}(N)$ is continuous on the above open set.

Remark 8. "Preference announcement" over the feasible set of the grand coalition does not imply that the same property holds over the (more restricted) feasible set of a smaller coalition. The continuity of $v^{*}(S)$, for $S \neq N$, therefore, does not follow from proposition 6 . 


\section{REFERENCES}

Allen, B. (1992): "Incentives in Market Games with Asymmetric Information: the Core," CORE Discussion Paper 9221, Université Catholique de Louvain.

(1993): "Market Games with Asymmetric Information: Verification and the Publicly Predictable Core," Hitotsubashi Journal of Economics, 32, 101-122.

_ (1994): "Incentives in Market Games with Asymmetric Information: Approximate NTU Cores in Large Economies," in Social Choice, Welfare and Ethics, ed. by W. Barnett, H. Moulin, M. Salles and N. Schofield. Cambridge: Cambridge University Press.

Arrow, K. (1979): "The Property Rights Doctrine and Demand Revelation under Incomplete Information," in Economics and Public Welfare, ed. by M. Boskin. New York: Academic Press.

Crémer, J., And R. MCLean (1985): "Optimal Selling Strategies under Uncertainty for a Discriminating Monopolist when Demands are Interdependent," Econometrica, 53, 345-361.

D'Aspremont, C., J. Crémer, and L.-A. GÉrard-Varet (1990): "Incentives and the Existence of Pareto-Optimal Revelation mechanisms," Journal of Economic Theory, 51, 233-254.

— (1992): "Mécanismes Bayésiens Incitatifs: un survol informel de quelques résultats récents," Annales d'Économie et de Statistique, 25/26, 151-164.

- (1995): "Correlation, Independence and Bayesian Incentives," mimeo.

D'Aspremont, C., And L.-A. GÉrard-Varet (1979): "Incentives and Incomplete Information," Journal of Public Economics, 11, 25-45.

_ (1982): "Bayesian Incentive Compatible Beliefs," Journal of Mathematical Economics, 10, 83-103.

Forges, F., And E. Minelli (1999): "A Note on the Incentive Compatible Core," Journal of Economic Theory, 98, 179-188.

Forges, F., E. Minelli, And R. Vohra (2001): "Incentives and the Core of an Exchange Economy: A Survey," CORE Discussion Paper 2001/43, Université Catholique de Louvain.

FudenberG, D., And J. Tirole (1991): Game Theory. Cambridge: MIT Press.

Green, J., And J.-J Laffont (1979): Incentives in Public Decision Making. Amsterdam: North Holland.

Groves, T. (1973): "Incentives in Teams," Econometrica, 41, 
617-631.

Groves, T., And M. Loeb (1975): "Incentives and Public Inputs," Journal of Public Economics, 4, 211-226.

Hildenbrand, W., AND A. Kirman (1988): Equilibrium Analysis: Variations on Themes by Edgeworth and Walras. Amsterdam: North-Holland.

Holmström, B., AND R. MYerson (1983): "Efficient and Durable Decision Rules with Incomplete Information," Econometrica, 51, 1799-1819.

Hurwicz, L., E. Maskin, And A. Postlewaite (1995): "Feasible Nash Implementation of Social Choice Rules when the Designer does not know Endowments or Production Sets," in The Economics of Informational Decentralization: Complexity, Efficiency and Stability, ed. by J.O. Ledyard. Amsterdam: Kluwer Academic Publishers, 367-433.

ICHIISHI, T., AND A. IDZIK (1996): "Bayesian Cooperative Choice of Strategies," International Journal of Game Theory, $25,455-473$.

Johnson, S., J. Pratt, And R. Zeckhauser (1990): "Efficiency despite Mutually Payoff-Relevant Private Information: the Finite Case," Econometrica, 58, 873-900.

Koutsougeras, L., And N. Yannelis (1993): "Incentive Compatibility and Information Superiority of the Core of an Economy with Differential Information," Economic Theory, 3, 195216.

Lee, D., And O. VoliJ (2000): "The Core of Economies with Asymmetric Information: An Axiomatic Approach," Journal of Mathematical Economics, forthcoming.

Mclean, R., AND A. Postlewaite (1999): "Informational Size and Incentive Compatibility," mimeo.

- (2000): "Informational Size, Incentive Compatibility and the Core of a Game with Incomplete Information," mimeo.

Milgrom, R., And J. Roberts (1992): Economics, Organization and Management. New Jersey: Prentice-Hall.

Myerson, R. (1984): "Cooperative Games with Incomplete Information," International Journal of Game Theory, 13, 69-96.

Myerson, R., And M. Satterthwaite (1983): "Efficient Mechanisms for Bilateral Trading," Journal of Economic Theory, 23, 265-281.

Prescott, E., And R. Townsend (1984): "General Competitive Analysis in an Economy with Private Information," International Economic Review, 25, 1-20. 
ScArf, H. (1967): "The Core of an N-Person Game," Econometrica, 35, 50-69.

Shapley, L., And M. Shubik (1969): "On Market Games," Journal of Economic Theory, 1, 9-25.

Shubik, M. (1982): Game Theory in the Social Sciences. M.I.T. Press.

Vohra, R. (1999): "Incomplete Information, Incentive Compatibility and the Core," Journal of Economic Theory, 86, 123-147.

VoliJ, O. (2000): "Communication, Credible Improvements and the Core of an Economy with Asymmetric Information," International Journal of Game Theory, 29, 63-79.

WiLSON, R. (1978): "Information, Efficiency and the Core of an Economy," Econometrica, 46, 807-816.

Williams, S. (1999): "A Characterization of Efficient, Bayesian Incentive Compatible Mechanisms," Economic Theory, 14, $155-180$.

YAnNelis, N. (1991): "The Core of an Economy with Differential Information," Economic Theory, 1, 183-198. 